\title{
Non-unique solutions of the Navier-Stokes equations for the Karman swirling flow
}

\author{
P. J. ZANDBERGEN and D. DIJKSTRA \\ Department of Applied Mathematics, Twente University of Technology, Enschede, The Netherlands \\ (Received November 17, 1976)
}

\begin{abstract}
SUMMARY
In this paper it is shown numerically that axially-symmetric solutions of the Navier-Stokes equations, which describe the rotating flow above a disk which is itself rotating, are non-unique. The numerical techniques designed to calculate such solutions with a high power of resolution are given. Especially the behaviour in and around the first branching point is considered. It is found that for $s=-0.16054$ two branches coincide. The second branch has been almost completely calculated. It ranges back to positive values of $s$.
\end{abstract}

\section{Introduction}

The problem of a rotating fluid above an infinite disk which is itself rotating has received considerable attention both from a theoretical and a computational point of view. In the case of axially symmetric flow, the Navier-Stokes equations can be reduced to a set of two ordinary non-linear differential equations with appropriate boundary conditions. For various values of the ratio $s$ of the angular velocity of the fluid and of the disk, solutions have been obtained, albeit sometimes with considerable effort. However for values of $s$ in the range $-0.160>s>$ -1.4351 it appeared to be impossible to find solutions, at least when no suction through the plate is applied.

The first to observe that for negative values of $s$ problems might occur were Rogers and Lance in 1960 [1]. Evans in 1969 [2], Ockendon in 1972 [3] and Bodonyi in 1975 [4] have made more detailed studies of the situation, and it is now clear that for $s=-1.4351$ the solution of the equations becomes singular. However at the other edge (i.e. near $s=-0.160$ ) the situation is much more mysterious. Evans claims, somewhat cryptically, that the numerical method did not fail but that the trouble was entirely due to large gradients giving rise to a very small integration stepsize; whereas Bodonyi reports that his numerical method fails to converge for no apparent reason. An attempt to clarify the situation has been made by Weidman and Redekopp [5]. They use a series expansion around $s=0$. From their results they try to obtain the behaviour of the nearest singularity. They propose that it is a $\frac{4}{3}$ power singularity at $s=-0.154$. But the evidence they advance appears to be unconclusive.

Theoretical investigations consider either the case $s>0$, or they allow for suction through the plate. It is necessary in this case to mention the names of Hastings [6], McLeod [7], [8], [9], Bushell [10], Hartman [11] and Lan [12]. Especially the investigations of McLeod should be recalled, since he establishes a number of interesting facts about the solution. Moreover he has 
proved that a solution is not possible for $s=-1$ and zero suction. In his investigations on the existence of a solution [8], he used a kind of shooting technique which starts from the asymptotic values far from tine disk and relates those to the values at the disk.

The numerical analogue of that technique is a basic tool in the present investigation. By its application it will be shown that the situation for the point where the solution becomes critical can be completely resolved. In fact we will show numerically that at the critical point $s=$ -0.16054 two solution branches coincide. This conclusion is supported analytically in the Appendix by means of first-order perturbation theory near the critical point.

In Section 2 we present the differential equations and asymptotic formulae for the solutions as well as some other facts which are interesting in order to obtain a check on the computations. In Section 3 the numerical techniques will be presented, whereas in Section 4 we give a thorough discussion of the results. This also includes a comparison with those of [1], [2] and [5]. Finally we give some concluding remarks, containing lines for future work.

\section{The governing equations}

In a cylindrical corrdinate system $(r, \phi, z)$ the disk is the plane $z=0$, and the corresponding velocities are

$$
u=r \Omega f^{\prime}(x), \quad v=r \Omega g(x), \quad w=-2(v \Omega)^{\frac{1}{2}} f(x) .
$$

The angular velocity of the disk is $\Omega$ and $x=z(\Omega / v)^{\frac{1}{2}}$. A prime denotes differentiation with respect to $x \in[0, \infty]$. As has been shown (see for example Schlichting [13]), the Navier-Stokes equations reduce in this case to

$$
\begin{aligned}
& f^{\prime \prime \prime}+2 f f^{\prime \prime}=f^{\prime 2}+s^{2}-g^{2}, \\
& g^{\prime \prime}+2 f g^{\prime}=2 f^{\prime} g .
\end{aligned}
$$

For zero suction the appropriate boundary conditions are

$$
\begin{aligned}
& x=0: f=0, f^{\prime}=0, g=1, \\
& x=\infty: f^{\prime}=0, g=s,
\end{aligned}
$$

where $s \Omega$ is the angular velocity of the fluid at infinity. Since the asymptotic behaviour of the solution at infinity plays a fundamental role in our investigations we give here the necessary formulae. They were first given by Rogers and Lance [1] and later rigorously proved to be valid by McLeod [7]. They are, for $x \rightarrow \infty$,

$$
\begin{aligned}
& f \sim a+\mathrm{e}^{p x}\left\{\frac{b p+c q}{p^{2}+q^{2}} \sin q x+\frac{c p-b q}{p^{2}+q^{2}} \cos q x\right\}, \\
& f^{\prime} \sim \mathrm{e}^{p x}\{b \sin q x+c \cos q x\}, \\
& f^{\prime \prime} \sim \mathrm{e}^{p x}\{(b p-c q) \sin q x+(c p+b q) \cos q x\}, \\
& g \sim s+\mathrm{e}^{p x}\{c \sin q x-b \cos q x\},
\end{aligned}
$$




$$
g^{\prime} \sim \mathrm{e}^{p x}\{(c p+b q) \sin q x+(c q-b p) \cos q x\} .
$$

The relation between $a, p$ and $q$ is given as follows:

$$
p^{2}-q^{2}=-2 a p, \quad p q=-a q+s .
$$

The second-order corrections to be added to the approximations (2.5)-(2.6) are given by

$$
f_{2}^{\prime}=c_{f} \mathrm{e}^{2 p x}, \quad g_{2}=c_{g} \mathrm{e}^{2 p x},
$$

with similar relations for $f_{2}, f_{2}^{\prime \prime}$ and $g_{2}^{\prime}$. The constants $c$ are

$$
c_{f}=\frac{\left(b^{2}+c^{2}\right)\left(2 q^{2}-p^{2}\right)}{2\left\{\left(p^{2}+q^{2}\right)^{2}+s^{2}\right\}}, \quad c_{g}=\frac{s\left(b^{2}+c^{2}\right)\left(q^{2}-5 p^{2}\right)}{2\left(p^{2}+q^{2}\right)\left\{\left(p^{2}+q^{2}\right)^{2}+s^{2}\right\}} .
$$

In the course of our studies we will also make use of a different formulation of the problem. For this purpose we write for $s \neq 0$,

$$
\eta=\sigma x, \quad \sigma=\sqrt{|s|},
$$

and

$$
f^{(k)}(x)=\sigma^{k+1} F^{(k)}(\eta), \quad g^{(k)}(x)=s \sigma^{k} G^{(k)}(\eta) .
$$

The new system in terms of $\eta$ is

$$
\begin{aligned}
& F^{\prime \prime \prime}+2 F F^{\prime \prime}=F^{\prime 2}+1-G^{2}, \\
& G^{\prime \prime}+2 F G^{\prime}=2 F^{\prime} G .
\end{aligned}
$$

The boundary conditions in this case are

$$
F(0)=F^{\prime}(0)=0, G(0)=1 / s, \quad F^{\prime}(\infty)=0, G(\infty)=1 .
$$

The asymptotic behaviour for $\eta \rightarrow \infty$ reads ( $F$ and $G$ only):

$$
\begin{aligned}
& F \sim A+\mathrm{e}^{P \eta}\left\{\frac{B P+C Q}{P^{2}+Q^{2}} \sin Q \eta+\frac{C P-B Q}{P^{2}+Q^{2}} \cos Q \eta\right\}+\frac{C_{F}}{2 P} \mathrm{e}^{2 P \eta}, \\
& G \sim 1+\operatorname{sign}(s) \mathrm{e}^{P \eta}\{C \sin Q \eta-B \cos Q \eta\}+C_{G} \mathrm{e}^{2 P \eta},
\end{aligned}
$$

where

$$
P^{2}-Q^{2}=-2 A P, P Q=-A Q+\operatorname{sign}(s),
$$

and

$$
C_{F}=\frac{\left(B^{2}+C^{2}\right)\left(2 Q^{2}-P^{2}\right)}{2\left\{\left(P^{2}+Q^{2}\right)^{2}+1\right\}}, C_{G}=\frac{\left(B^{2}+C^{2}\right)\left(Q^{2}-5 P^{2}\right)}{2\left(P^{2}+Q^{2}\right)\left\{\left(P^{2}+Q^{2}\right)^{2}+1\right\}} .
$$


As will be clear, we can consider the boundary value problem (2.2)-(2.4) also as a problem where we make use of the asymptotic formulae and where we find a solution of the system (2.2)(2.3) such that the relations

$$
f(x=0 ; a, b, c)=0, f^{\prime}(x=0 ; a, b, c)=0, g(x=0 ; a, b, c)=1
$$

are satisfied.

The following important property was derived by McLeod [8], [9]

$$
f^{\prime \prime}(x) f^{\prime \prime \prime}(x)+g^{\prime}(x) g^{\prime \prime}(x)<0
$$

which means that the function $f^{\prime \prime 2}(x)+g^{\prime 2}(x)$ is strictly decreasing to zero at infinity. From this it can be derived that the value of $f^{\prime \prime}(0)$ is positive, while the value of $g^{\prime}(0)$ should be negative. Moreover $f(\infty)=a$ is bounded.

In order to have an independent check on the numerical calculations, it is useful to consider the following equations

$$
\begin{aligned}
& f^{\prime \prime 2}(0)-g^{\prime 2}(0)=\int_{0}^{\infty} 4 f\left(f^{\prime \prime 2}-g^{\prime 2}\right) d x \\
& f^{\prime \prime}(0) g^{\prime}(0)+\frac{2}{3} s^{3}-s^{2}+\frac{1}{3}=\int_{0}^{\infty} 4 f f^{\prime \prime} g^{\prime} d x .
\end{aligned}
$$

These results are derived by multiplying eqs. (2.2) and (2.3) by respectively $f^{\prime \prime}$ and $g^{\prime}$ or $g^{\prime}$ and $f^{\prime \prime}$, followed by subtraction and addition.

\section{The numerical approach}

In order to obtain solutions to the problem, two different numerical techniques have been used. The first method is a finite-difference technique applied to the problem formulation (2.2)-(2.4). This method uses central differencing and Newton iteration to solve the resulting set of nonlinear algebraic equations. The second method is a shooting technique which produces more accurate results than the finite-difference method. The equations are integrated inward from infinity down to the origin. This approach is of the initial-value type, so that iteration is necessary in order to satisfy the boundary conditions at the disk. Two versions of the shooting method have been used. The first one is based on the formulation (2.12)-(2.14) and the second one uses the original formulation (2.2)-(2.4).

\subsection{The finite difference method}

We rewrite the system (2.2)-(2.4) as follows

$$
\begin{aligned}
& f^{\prime}=u \\
& u^{\prime \prime}+2 f u^{\prime}-u^{2}+g^{2}=s^{2} \\
& g^{\prime \prime}+2 f g^{\prime}-2 u g=0
\end{aligned}
$$


with boundary conditions

$$
\begin{aligned}
& f(0)=u(0)=0, \quad g(0)=1, \\
& u(\infty)=0, \quad g(\infty)=s
\end{aligned}
$$

The boundary conditions (3.4b) are applied at a finite value $x=x_{m}$, i.e.,

$$
u\left(x_{m}\right)=0, \quad g\left(x_{m}\right)=s .
$$

The choice of $x_{m}$ depends on circumstances. For instance, the Rogers and Lance [1] solutions in the region $0 \leqq s \leqq 1$ can be produced working with $x_{m}=16$ while the new solutions which we have obtained require a much larger range. The largest value of $x_{m}$ in the present calculations with the finite difference method was 52 .

The mesh covering the range $0 \leqq x \leqq x_{m}$ is uniform with step size $h$ and mesh points $x_{j}$ given by

$$
h=x_{m} / N, x_{j}=h j, \quad j=0(1) N .
$$

All finite-difference calculations have been performed with step sizes $h=0.2,0.1$ and 0.05 .

Equation (3.1) is integrated by means of the trapezoidal rule and the equations for $u$ and $g$ are discretized by central differences. The system of equations for the quantities $f_{j}, u_{j}$ and $g_{j}$ reads

$$
\begin{aligned}
& f_{j}-f_{j-1}-\frac{h}{2}\left(u_{j-1}+u_{j}\right)=0, \quad j=1(1) N, \\
& \frac{1}{h^{2}}\left(u_{j+1}-2 u_{j}+u_{j-1}\right)+\frac{1}{h} f_{j}\left(u_{j+1}-u_{j-1}\right)-u_{j}^{2}+g_{j}^{2}=s^{2}, \quad j=1(1) N-1, \\
& \frac{1}{h^{2}}\left(g_{j+1}-2 g_{j}+g_{j-1}\right)+\frac{1}{h} f_{j}\left(g_{j+1}-g_{j-1}\right)-2 u_{j} g_{j}=0, \quad j=1(1) N-1 .
\end{aligned}
$$

This system of $3 N-2$ non-linear algebraic equations is solved by means of Newton iteration. If $n$ denotes the iteration index we set

$$
f_{j}^{(n+1)}=f_{j}^{(n)}+\delta f_{j}^{(n)}, u_{j}^{(n+1)}=u_{j}^{(n)}+\delta u_{j}^{(n)}, g_{j}^{(n+1)}=g_{j}^{(n)}+\delta g_{j}^{(n)}
$$

and substitute this into (3.6), neglecting terms which are quadratic in the quantities $\delta$. We then arrive at a system of equations which is linear in the Newton corrections $\delta$. By arranging the solution vector $V_{\delta}^{(n)}$ as follows,

$$
V_{\delta}^{(n)}=\left(\delta f_{1}^{(n)}, \delta g_{1}^{(n)}, \delta u_{1}^{(n)}, \ldots, \delta f_{N-1}^{(n)}, \delta g_{N-1}^{(n)}, \delta u_{N-1}^{(n)}, \delta f_{N}^{(n)}\right)
$$

it is found that the band width of the system is 7 and the matrix routine which solves the equations takes advantage of this property in an obvious way.

The iteration is ended if $\left\|V_{\delta}^{(n)}\right\|<10^{-6}$. In all cases where the solution is non-unique the procedure appears to converge to one solution or the other depending on the starting values 
used to initialize the iteration. The only exception is a small neighbourhood of the critical point as will be further explained in Sec. 4.1 .

The converged solution contains the following errors.

i) Round-off error, due to the limited machine accuracy.

ii) Truncation error, caused by the truncation of the iteration.

These errors are neglibly small as compared with

iii) Cut-off error, arising from the use of (3.4c) instead of (3.4b).

iv) Discretization error introduced by the discretization of the differential equations.

The cut-off error is made small by taking the upper bound $x_{m}$ of the integration range large enough. Error (iv) is reduced by means of Richardson extrapolation based on $O\left(h^{2}\right)$.

We conclude this subsection with some remarks concerning the accuracy and computing time of the finite-difference method.

Accuracy. The Richardson extrapolation considerably improves the accuracy of the results as shown in the following table for $f(\infty)$ at $s=0$.

TABLE 3.1

The effect of Richardson extrapolation on the results obtained with $x_{m}=24$

\begin{tabular}{llll}
\hline$h$ & $2 f(\infty)$ & extrapolated & exact value \\
\hline 0.2 & 0.881101 & - & \\
0.1 & 0.883626 & 0.884468 & \\
0.05 & 0.884262 & 0.884474 & 0.884474 \\
\hline
\end{tabular}

We infer that the finite-difference method can produce 5-6 significant digits for $f(\infty)$. However, the values for $f^{\prime \prime}(0)$ and $g^{\prime}(0)$ are less accurate since they are obtained by numerical differentiation.

Computing time. A full run through the range $0 \leqq s \leqq 1$ with $s=0(0.1) 1$ and $x_{m}=16$ takes 12 sec. for $h=0.2$ on a Dec 10 computer. The time required for $h=0.1$ is twice as large.

\subsection{The shooting methods}

We first describe the method which has been used to pass the critical region. The second method can be applied to the remaining part of the solution curve.

With $F(\infty)=A$ prescribed the equations (2.12) and (2.13) are integrated from $\eta=\eta_{m}$ down to $\eta=0$. The integration is performed with a routine called Diffsys developed by Bulirsch and Stoer [14]. The initial values of $F, F^{\prime}, F^{\prime \prime}, G$ and $G^{\prime}$ at $\eta=\eta_{m}$ are obtained from the second order asymptotic approximations (2.15) (2.16) with guessed values for $B$ and $C$. Note that the error in the initial values at $\eta=\eta_{m}$ if of the order $\exp \left(3 P \eta_{m}\right)$ as compared with $\exp \left(P \eta_{m}\right)$ in the method used by Evans [2]. The end point of the integration is $\eta=0$ where both $F$ and $F^{\prime}$ should vanish. With a fixed value of $F(\infty)=A$, we consider the quantities $F(0)$ and $F^{\prime}(0)$ as functions of $B$ and $C$. These parameters are iteratively determined in such a way that the boundary conditions

$$
F(\eta=0 ; B, C)=F^{\prime}(\eta=0 ; B, C)=0
$$


are satisfied. The iteration on $B$ and $C$ always appears to converge, provided that the initial values are selected properly. The value of $s$ follows from the converged solution, viz.

$$
s=1 / G(0)
$$

see eq. (2.14). Once the value of $s$ has been determined, the transformation (2.10)-(2.11) can be used to find the relevant quantities in terms of the formulation (2.2)-(2.3). Note that the solution automatically merges with the asymptotic behaviour (2.15)-(2.16) for large $\eta$. In fact this property was used as a check on the calculations. Further, the checks (2.20)-(2.22) when translated in terms of the present variables - were always found to be satisfied within the accuracy of the calculation.

The idea to prescribe $F(\infty)=A$ instead of $s$ in this method appears to be crucial when passing the point where $s=s_{c r}$. The difficulty at $s=s_{c r}$ is caused by the vanishing of the derivative $d s / d A$, see Section 4 and the Appendix.

The present method fails at $s=0$ (see eq. (2.10)). To remove this failure as well as to obtain results which can be compared with known results from the literature, a modified shooting method was developed. In this modified shooting method we use the formulation (2.2)-(2.4) with $s$ prescribed. The shooting technique is the same as before (with second order asymptotics) on the understanding that now an iteration is performed on the parameters $a, b$ and $c$, in order to satisfy the boundary conditions (2.19). For $s \neq 0$ and $s \neq s_{c r}$ the two shooting methods are found to produce the same results.

Finally, some remarks are made concerning accuracy and computing time.

Accuracy. With the shooting methods a high degree of accuracy can be obtained (working in double precision). This is illustrated by a comparison with the results of Weidman and Redekopp [5] at $s=0$.

TABLE 3.2

A comparison with the results of Weidman and Redekopp at $s=0$

\begin{tabular}{llll}
\hline & $2 f(\infty)$ & $f^{\prime \prime}(0)$ & $-g^{\prime}(0)$ \\
\hline present & 0.8844741102096 & 0.5102326188673 & 0.6159220143994 \\
ref. [5] & 0.88447411021 & 0.5102326188673 & 0.6159220143993 \\
\hline
\end{tabular}

(For a further comparison with the results of Weidman and Redekopp see Section 4.3.)

Computing time. This depends strongly on the accuracy of the calculation, the choice of the starting point $\eta_{m}$ (which should be sufficiently large) as well as the quality of the starting values for $B$ and $C$. Per value of $F(\infty)$ the time varies from 10 to 60 seconds on a Dec-10 computer.

\section{Discussion of the results}

In this section we will subsequently discuss the following items. In Subsection 4.1 we will give an outline of the way followed to find the solutions. In Subsection 4.2 we present full details of 
actual numerical results, while in Subsection 4.3 a comparison will be made with the results of Rogers and Lance [1], Evans [2] and Weidman and Redekopp [5].

\subsection{The construction of the solutions}

In the first place a start was made with the finite-difference method as described in Section 3.1. It was concluded that for $s \approx-0.16$ the method failed to converge, but also that no sign of divergence was apparent. From the successive iteration steps it seemed that the solution would converge for about 3 or 4 steps and then jump to another level and converge again for 3 or 4 steps. This then gave rise to the assumption that we had to do with two solutions close together, where the iteration process would jump from one solution to the other without having enough power of distinction between the two solutions. This assumption could be proved valid by changing the range of the calculation from the usual one used by Evans, i.e. $0 \leqq x \leqq 12$, to a range with endpoints $x_{m}=16$ or $x_{m}=24$ and carefully choosing the initial profile of the iteration. In this way it appeared possible to identify two apparently quite distinct solutions for the same value of $s$. This has been illustrated in Figure 1, where $f^{\prime}$ has been given together with the corresponding value of $f(\infty)$. It will be clear that, although we now had evidence of the non-uniqueness of the solutions, we by no means had an accurate numerical solution of the original problem. In order to surmount this difficulty use was made of the method described in Section 3.2.

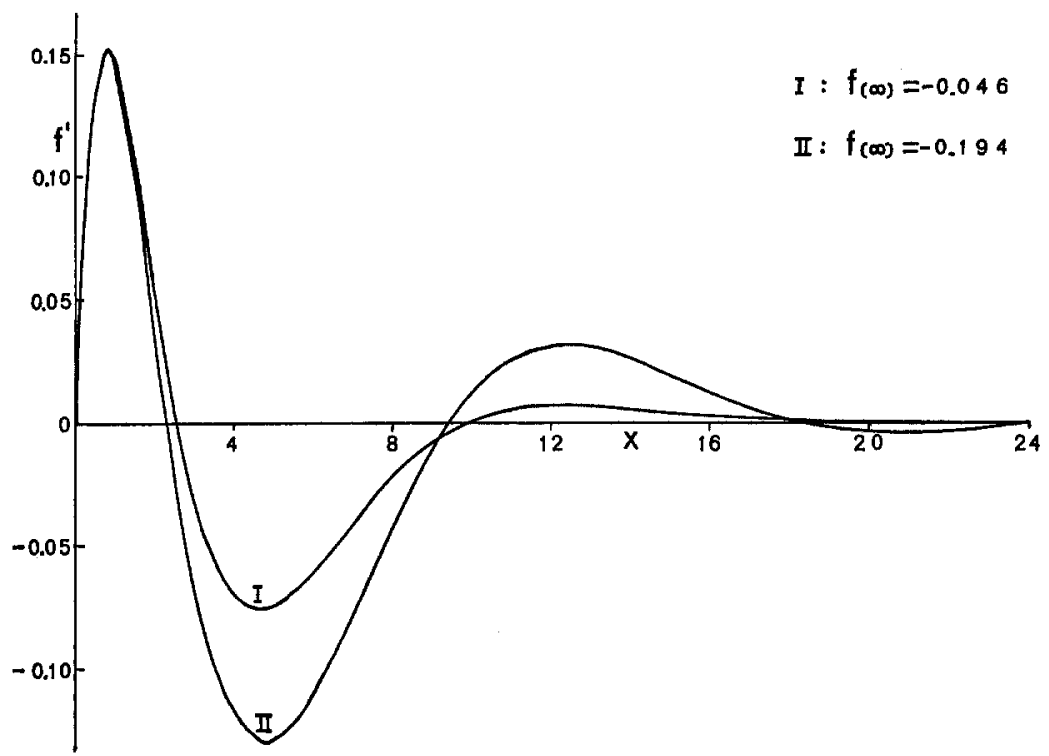

Figure 1. Two solutions $f^{\prime}(x)$ at $s=-0.1575$ as constructed with the finite difference method $\left(x_{m}=24, h=0.1\right)$.

This method had proved its power already in a study of the Bödewadt problem and also here it gave the solution to our problem in a very easy and accurate way. This accuracy depends on three factors. Firstly, infinity is taken into account in the right way and such that the accuracy can be controlled. In the second place use is made of a very accurate and fast integration routine for the differential equations, and thirdly it appears that the stability of inverse shooting is much better than trying to find the solution by shooting from $\eta=0$. This can be explained in part by 
the fact that the gradients with respect to $s$ of the determining quantities at infinity are orders of magnitude larger than those at the origin. Apart from accuracy an additional advantage of the inverse shooting technique is the fact that it is very easy to prescribe $F(\infty)$, leaving the quantity $s$ as an output of the calculation, see eq. (2.14). Naturally, the question arose to find the second branch of the solution and to investigate its precise behaviour. Where will it end and how will it come to an end?

In this paper we have not solved the last questions. The second branch is constructed, which bends backwards from $s=s_{c r}=-0.1605387613$ to positive values of $s$. We have strong evidence that there exists a second branching point near $s=+0.09$. $^{\star}$

In order to construct the second branch, use was made of the various computational schemes

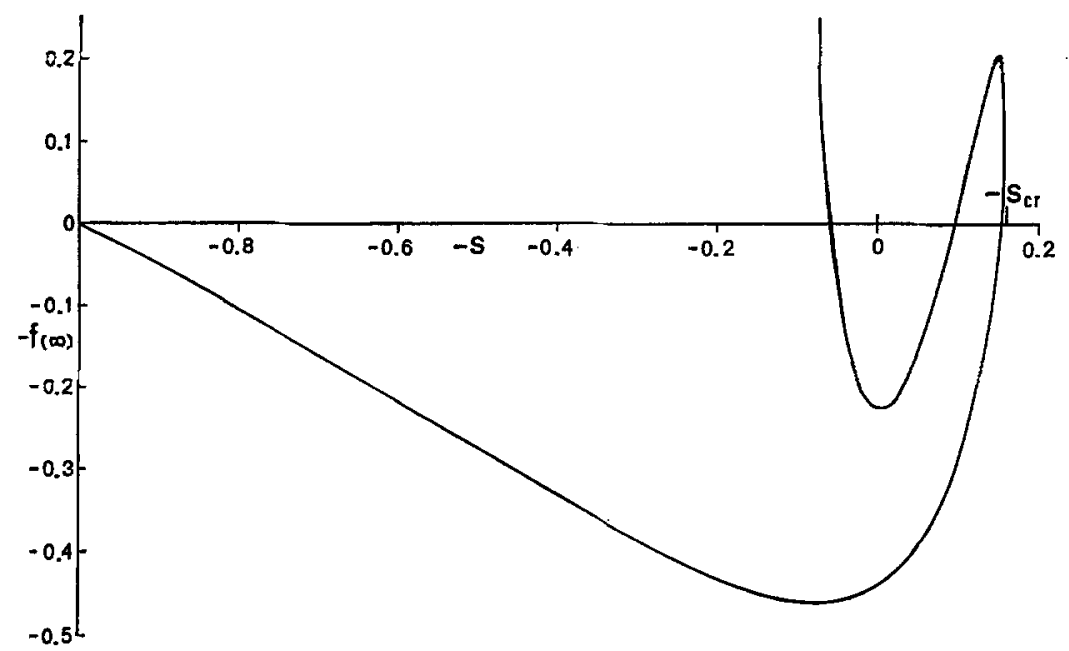

Figure 2. The vertical velocity at infinity as a function of $-s$.

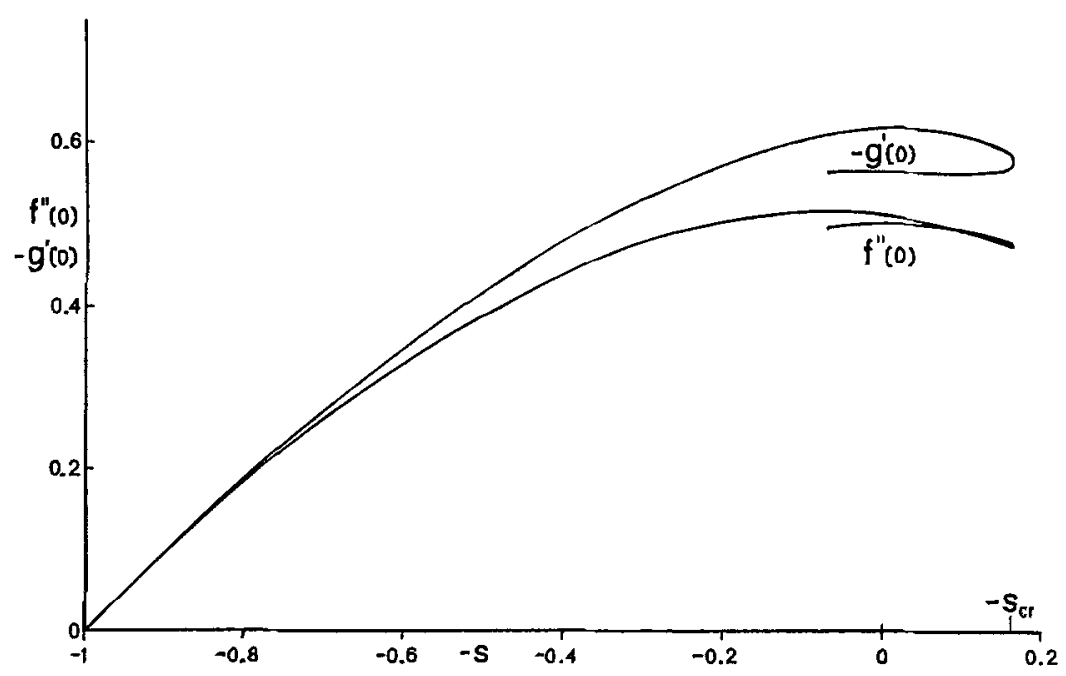

Figure 3. The quantities $f^{\prime \prime}(0)$ and $-g^{\prime}(0)$ as a function of $-s$.

$\star$ Note added in proof: Recent calculations produced for the value of the second branching point: $s=0.07452563$. 
as discussed in the previous section. In regions away from the branching points the difference scheme of Section 3.1 was used as a means to obtain basic information about the behaviour of the solution. Then afterwards the solution was calculated to full accuracy by using the shooting methods as described in Section 3.2. Away from the branching points the version with $s$ prescribed was used, while in the neighbourhood of the critical points the method with $F(\infty)$ prescribed was applied.

\subsection{Results}

The perhaps most important result of the present investigation is contained in Fig. 2 where we give the quantity $f(\infty)$ as a function of $s$ for the first and second branch. It is evident that - near

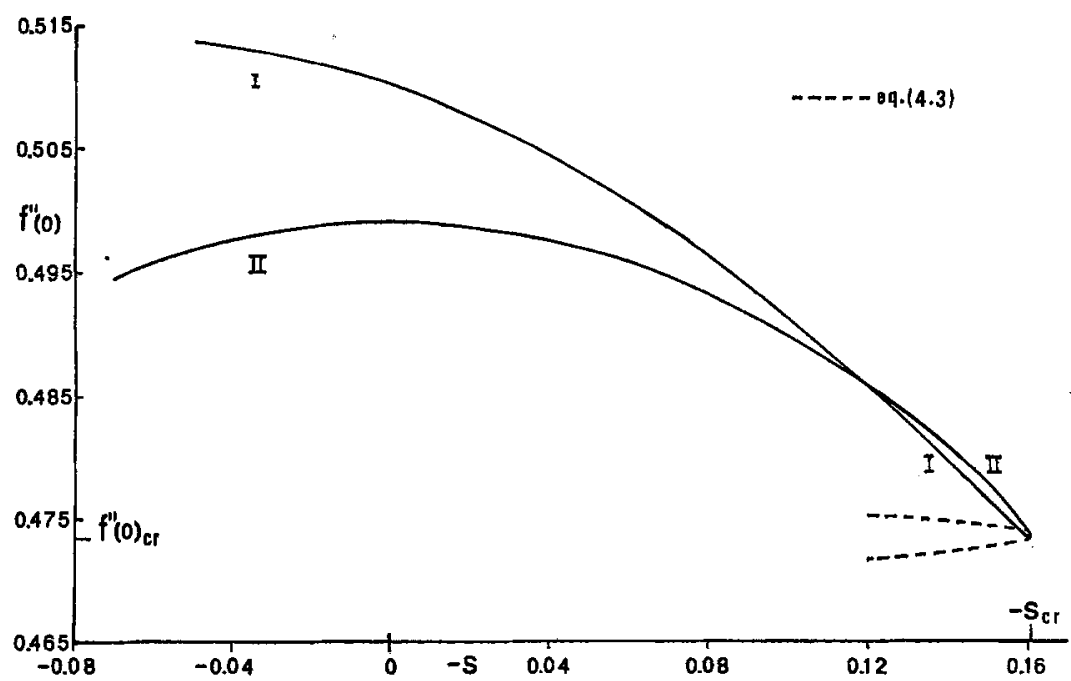

Figure 4a. The quantity $f^{\prime \prime}(0)$ as a function of $-s$ (detail of fig. 3 ).

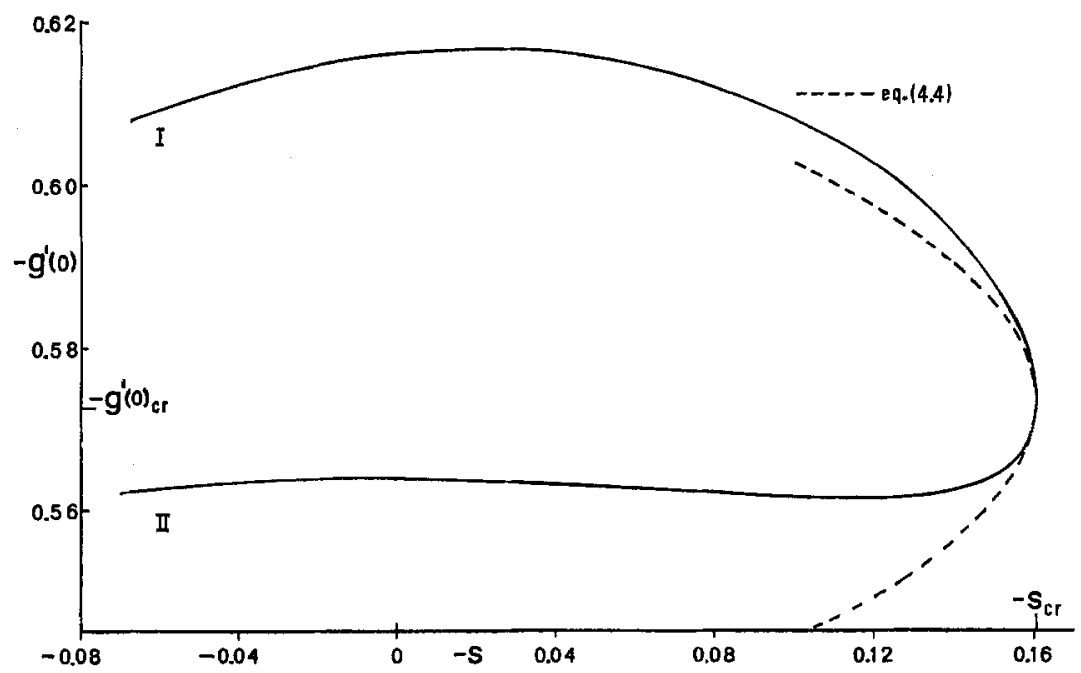

Figure 4 b. The quantity $-g^{\prime}(0)$ as a function of $-s$ (detail of fig. 3). 
infinity - these solutions are quite different for the different branches of the solution. As will be evident from Figs. $3,4 \mathrm{a}$ and $4 \mathrm{~b}$ this is not the case for the quantities $f^{\prime \prime}(0)$ and $g^{\prime}(0)$ which vary only slightly with $s$. This seems to us one of the reasons why it is so difficult to obtain results by shooting from $x=0$ as has been done by Rogers and Lance [1] and also in part by Evans [2].

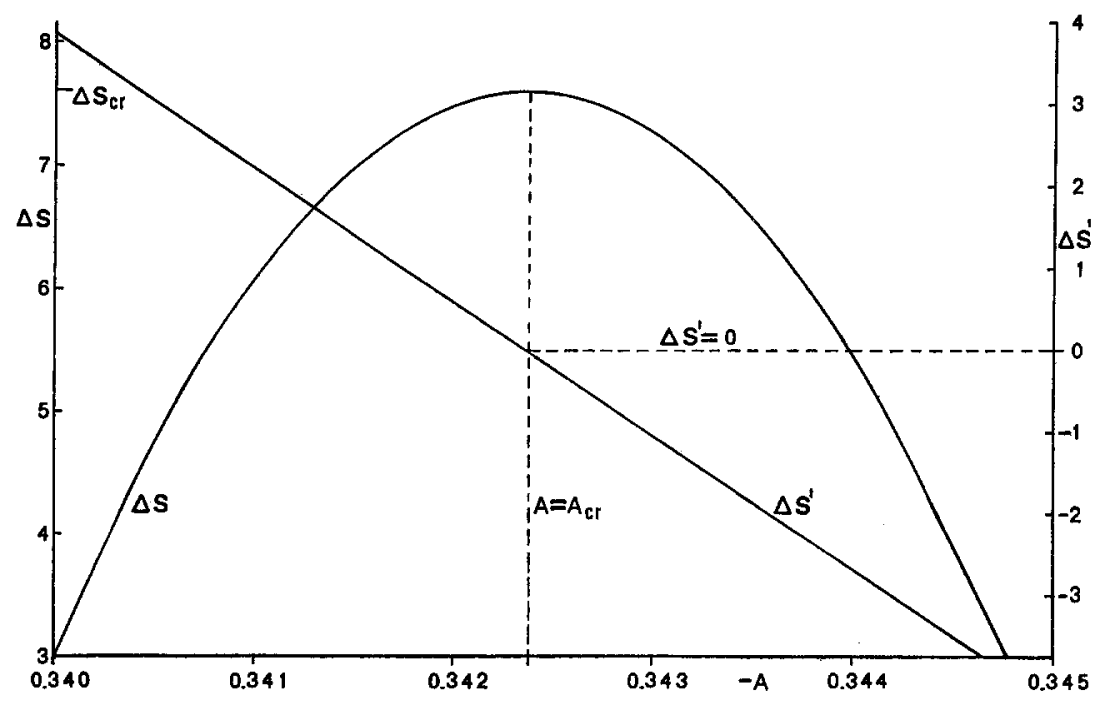

Figure 5a. The quantities $s$ and $d s / d A$ as a function of $A=F(\infty)$ near the critical point. $\Delta s=-10^{7}(0.160538+s)$; $\Delta s^{\prime}=10^{4} d s / d A$.

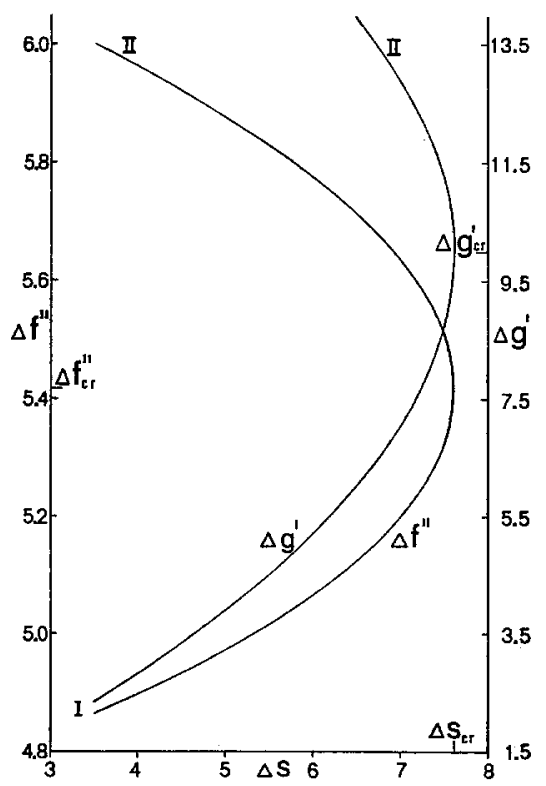

Figure 5b. Variation of $f^{\prime \prime}(0)$ and $g^{\prime}(0)$ with $s$ near the critical point. $\Delta s=-10^{7}(s+0.160538) ; \Delta f^{\prime \prime}=10^{5}\left(f^{\prime \prime}(0)\right.$ $-0.4735) ; \Delta g^{\prime}=10^{5}\left(g^{\prime}(0)+0.5732\right)$. 
TABLE 4.1

Results for the first solution branch (I)

\begin{tabular}{llll}
\hline$s$ & $2 f(\infty)$ & $f^{\prime \prime}(0)$ & $-g^{\prime}(0)$ \\
\hline 1.0 & 0 & 0 & 0 \\
0.9 & 0.10200741 & 0.09593565 & 0.09695077 \\
0.8 & 0.20800654 & 0.18346935 & 0.18759077 \\
0.7 & 0.31778953 & 0.26215136 & 0.27156095 \\
0.6 & 0.43077148 & 0.33147015 & 0.34843403 \\
0.5 & 0.54568250 & 0.39083916 & 0.41768942 \\
0.4 & 0.65998069 & 0.43957985 & 0.47867327 \\
0.3 & 0.76866766 & 0.47690044 & 0.53053067 \\
0.2 & 0.86169898 & 0.50187028 & 0.57208036 \\
0.15 & 0.89642794 & 0.50938861 & 0.58847271 \\
0.10 & 0.91761139 & 0.51339584 & 0.60155285 \\
0.05 & 0.91781860 & 0.51373101 & 0.61090263 \\
0 & 0.88447411 & 0.51023262 & 0.61592201 \\
-0.025 & 0.84862988 & 0.50700020 & 0.61653321 \\
-0.050 & 0.79413093 & 0.50276189 & 0.61565919 \\
-0.075 & 0.71359612 & 0.49751628 & 0.61304592 \\
-0.100 & 0.59450856 & 0.49128369 & 0.60828605 \\
-0.125 & 0.41179774 & 0.48413876 & 0.60058809 \\
-0.150 & 0.08941846 & 0.47636622 & 0.58746927 \\
-0.1525 & 0.03881348 & 0.47559507 & 0.58548370 \\
-0.1550 & -0.01990371 & 0.47484234 & 0.58319556 \\
-0.1575 & -0.09236631 & 0.47412746 & 0.58038111 \\
\hline
\end{tabular}

TABLE 4.2

Results for the second solution branch (II)

\begin{tabular}{lrll}
\hline$s$ & \multicolumn{1}{l}{$2 f(\infty)$} & $f^{\prime \prime}(0)$ & $-g^{\prime}(0)$ \\
\hline 0.07 & -0.31733586 & 0.49462745 & 0.56232679 \\
0.06 & -0.06011790 & 0.49580605 & 0.56266423 \\
0.04 & 0.24862538 & 0.49760889 & 0.56324042 \\
0.02 & 0.39569075 & 0.49868508 & 0.56361641 \\
0 & 0.44772333 & 0.49904295 & 0.56374644 \\
-0.025 & 0.42453859 & 0.49848359 & 0.56354428 \\
-0.050 & 0.32997008 & 0.49679910 & 0.56297300 \\
-0.075 & 0.17964509 & 0.49396763 & 0.56215245 \\
-0.100 & -0.01446720 & 0.48994137 & 0.56134736 \\
-0.125 & -0.23285212 & 0.48461328 & 0.56118094 \\
-0.150 & -0.40472151 & 0.47765325 & 0.56392059 \\
-0.1525 & -0.40840854 & 0.47682370 & 0.56472004 \\
-0.1550 & -0.40472036 & 0.47595188 & 0.56579413 \\
-0.1575 & -0.38794265 & 0.47501806 & 0.56736644 \\
\hline
\end{tabular}


TABLE 4.3a

Results for both branches near the critical point.

\begin{tabular}{lllll}
\hline$-A$ & $-s$ & $-f(\infty)$ & $f^{\prime \prime}(0)$ & $-g^{\prime}(0)$ \\
\hline 0 & 0.1542001134 & 0 & 0.4750803572 & 0.5839697645 \\
0.05 & 0.1557387474 & 0.0197318744 & 0.4746257207 & 0.5824356418 \\
0.10 & 0.1570965436 & 0.0396354063 & 0.4742386727 & 0.5808899655 \\
0.15 & 0.1582621396 & 0.0596732615 & 0.4739242956 & 0.5793308932 \\
0.20 & 0.1592195757 & 0.0798046554 & 0.4736891643 & 0.5777548562 \\
0.25 & 0.1599454450 & 0.0999829501 & 0.4735423386 & 0.5761556910 \\
0.30 & 0.1604035790 & 0.1201512468 & 0.4734972040 & 0.5745229913 \\
0.35 & 0.1605339313 & 0.1402334004 & 0.4735752972 & 0.5728386247 \\
0.40 & 0.1602252434 & 0.1601125821 & 0.4738156564 & 0.5710681012 \\
0.45 & 0.1592274505 & 0.1795649151 & 0.4743045866 & 0.5691327354 \\
0.50 & 0.1566488901 & 0.1978944732 & 0.4753451903 & 0.5667486507 \\
\hline
\end{tabular}

TABLE $4.3 \mathrm{~b}$

Values of $s, f^{\prime \prime}(0)$ and $g^{\prime}(0)$ for a given value of $A=F(\infty) ; f(\infty)=F(\infty) \sqrt{-s}$

\begin{tabular}{llll}
\hline$-A$ & $-s$ & $f^{\prime \prime}(0)$ & $-g^{\prime}(0)$ \\
\hline 0.325 & 0.1605149552 & 0.4735189969 & 0.5736888721 \\
0.330 & 0.1605265647 & 0.4735273479 & 0.5735202436 \\
0.335 & 0.1605343852 & 0.4735371121 & 0.5733509413 \\
0.340 & 0.1605383027 & 0.4735483293 & 0.5731809298 \\
0.3425 & 0.1605387600 & 0.4735544961 & 0.5730956464 \\
0.345 & 0.1605381951 & 0.4735610424 & 0.5730101712 \\
0.350 & 0.1605339313 & 0.4735752972 & 0.5728386247 \\
0.355 & 0.1605253705 & 0.4735911427 & 0.5726662463 \\
0.360 & 0.1605123612 & 0.4736086318 & 0.5724929887 \\
0.365 & 0.1604947394 & 0.4736278216 & 0.5723188007 \\
\hline
\end{tabular}

TABLE $4.3 \mathrm{c}$

Derivatives of relevant quantities, obtained by numerical differentiation in Table 4.3b.

\begin{tabular}{lllll}
\hline$-A$ & $10^{3} d s / d A$ & $10 d^{2} s / d A^{2}$ & $-10^{3} d f^{\prime \prime}(0) / d A$ & $-10^{2} d g^{\prime}(0) / d A$ \\
\hline 0.330 & 1.943 & 1.516 & 1.812 & 3.379 \\
0.335 & 1.174 & 1.561 & 2.098 & 3.393 \\
0.340 & 0.381 & 1.610 & 2.393 & 3.408 \\
0.3425 & -0.022 & 1.636 & 2.543 & 3.415 \\
0.345 & -0.437 & 1.662 & 2.697 & 3.423 \\
0.350 & -1.282 & 1.719 & 3.010 & 3.439 \\
0.355 & -2.157 & 1.779 & 3.333 & 3.456 \\
0.360 & -3.063 & 1.845 & 3.668 & 3.474 \\
\hline
\end{tabular}


TABLE $4.4 \mathrm{a}$

Values of $s, F^{\prime \prime}(0)$ and $G^{\prime}(0)$ for a given value of $A=F(\infty)$ near the critical point.

\begin{tabular}{llll}
\hline$-A$ & $-S$ & $F^{\prime \prime}(0)$ & $G^{\prime}(0)$ \\
\hline 0.3400 & 0.1605383027 & 7.362008391 & 8.910944361 \\
0.3405 & 0.1605384752 & 7.362015235 & 8.910665066 \\
0.3410 & 0.1605386073 & 7.362025089 & 8.910389017 \\
0.3415 & 0.1605386988 & 7.362037962 & 8.910116222 \\
0.3420 & 0.1605387498 & 7.362053865 & 8.909846692 \\
0.3425 & 0.1605387600 & 7.362072807 & 8.909580436 \\
0.3430 & 0.1605387293 & 7.362094797 & 8.909317465 \\
0.3435 & 0.1605386576 & 7.362119845 & 8.909057788 \\
0.3440 & 0.1605385448 & 7.362147962 & 8.908801416 \\
0.3445 & 0.1605383906 & 7.362179157 & 8.908548359 \\
0.3450 & 0.1605381951 & 7.362213440 & 8.908298626 \\
\hline
\end{tabular}

TABLE 4.4b

Derivatives of relevant quantities obtained with eqs. (A.3)-(A.6).

\begin{tabular}{lllll}
\hline$-A$ & $\begin{array}{l}-10^{3} s^{2} G_{1}(0)= \\
=10^{3} d s / d A\end{array}$ & $\begin{array}{l}10 d^{2} s / d A^{2} \\
(\mathrm{num} .)\end{array}$ & $\begin{array}{l}-10^{2} F_{1}^{\prime \prime}(0)= \\
=-10^{2} d F^{\prime \prime}(0) / d A\end{array}$ & $\begin{array}{l}10 G_{1}^{\prime}(0)= \\
=10 d G^{\prime}(0) / d A\end{array}$ \\
\hline 0.3400 & 0.385197 & - & 1.068365 & 5.618286 \\
0.3405 & 0.304583 & 1.6148 & 1.669456 & 5.553478 \\
0.3410 & 0.223714 & 1.6199 & 2.272436 & 5.488477 \\
0.3415 & 0.142589 & 1.6251 & 2.877319 & 5.423282 \\
0.3420 & 0.061205 & 1.6303 & 3.484120 & 5.357890 \\
0.3425 & -0.020438 & 1.6355 & 4.092855 & 5.292301 \\
0.3430 & -0.102343 & 1.6407 & 4.703537 & 5.226512 \\
0.3435 & -0.184511 & 1.6460 & 5.316183 & 5.160523 \\
0.3440 & -0.266946 & 1.6514 & 5.930806 & 5.094331 \\
0.3445 & -0.349647 & 1.6567 & 6.547424 & 5.027936 \\
0.3450 & -0.432618 & - & 7.166051 & 4.961335 \\
\hline
\end{tabular}

In Tables 4.1 and 4.2 we have presented accurate values of the relevant quantities for different values of $s$ along both branches. In Tables $4.3 \mathrm{a}, 4.3 \mathrm{~b}$ and $4.3 \mathrm{c}$ more detailed results are presented for the surrounding of the first branching point. The precise location of this critical point has been calculated from the results in Tables $4.4 \mathrm{a}$ and $4.4 \mathrm{~b}$ which have been obtained by using an additional set of perturbation equations as given in the Appendix. A graphical representation of these results is given in Figs. 5a and 5b. The curve in Fig. 5a can be accurately represented by means of the parabola

$$
s=-0.1605387613+0.08171(A+0.34237498)^{2}, \quad A=F(\infty) .
$$

Inversion of the result (4.1) yields in terms of $a=f(\infty)$ the approximation

$$
f(\infty)=-0.13718037 \pm 1.402(s+0.1605387613)^{\frac{1}{2}}
$$


where the upper sign corresponds with branch I of the solution and the lower sign with branch II. Similarly, the derivatives at the origin are found to be

$$
\begin{aligned}
& f^{\prime \prime}(0)=0.47355418 \mp 0.008866(s+0.1605387613)^{\frac{1}{2}}, \\
& g^{\prime}(0)=-0.57309992 \mp 0.1194(s+0.1605387613)^{\frac{1}{2}} .
\end{aligned}
$$

It will be clear that these approximations are valid only in the asymptotic sense $s \rightarrow s_{c r}$. Approximations with a larger range of validity will be considered in Subsection 4.3.

What now are the most significant differences in the results for the same value of $s$ ? We have illustrated this in Figs. 6a, $\mathrm{b}$ and $\mathrm{c}$ for $s=0$, which is the original von Karman case.

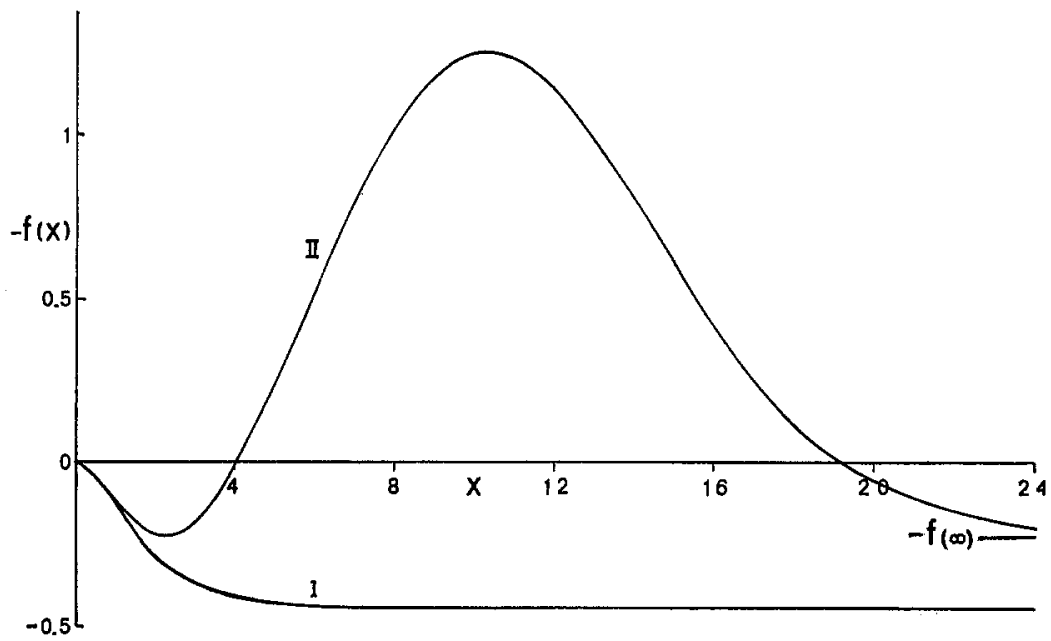

Figure 6a. The two vertical velocities for $s=0$.

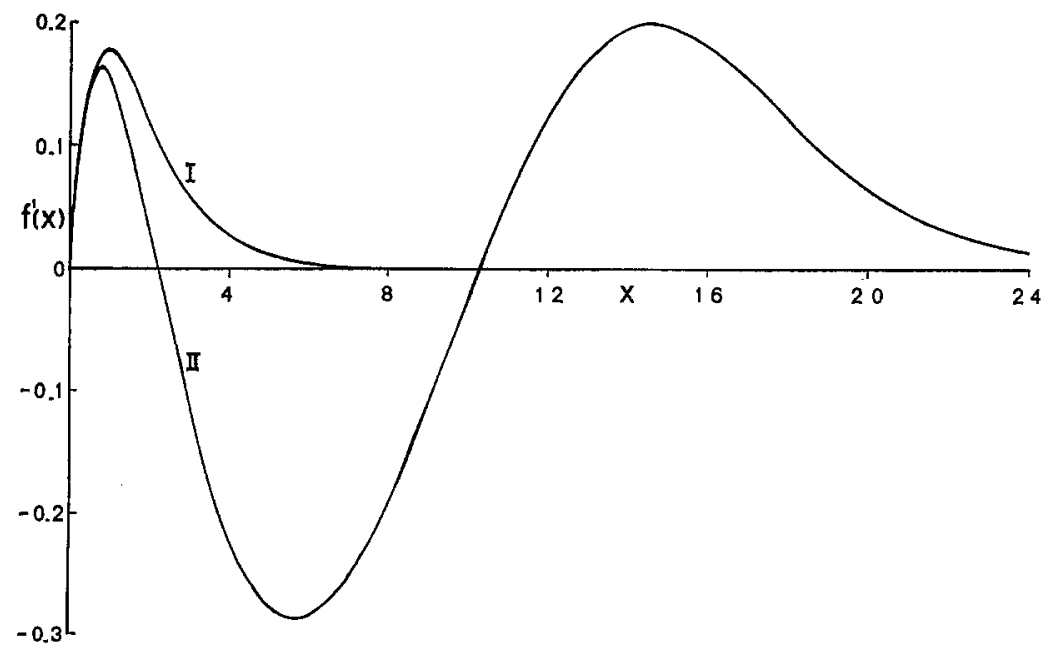

Figure $6 \mathrm{~b}$. The two radial velocities for $s=0$. 


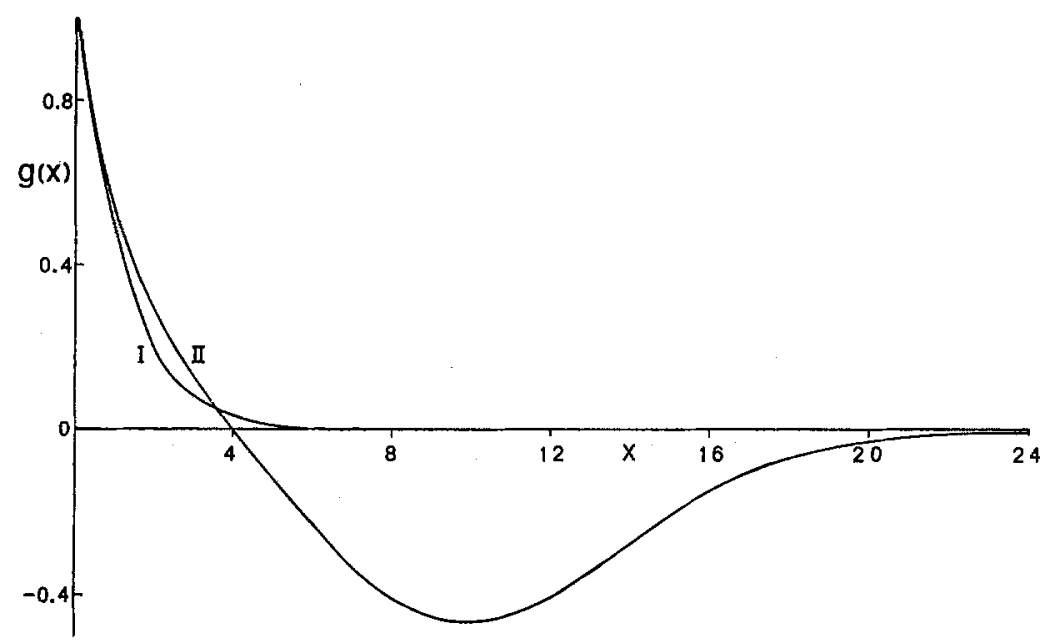

Figure $6 \mathrm{c}$. The two tangential velocities for $s=0$.

The already known solution is in fact a simple boundary layer type flow. The second solution, however is totally different away from the disk. The von Karman boundary layer again is present, nearly unperturbed, but now a second layer is present with strong counter rotating velocities together with large vertical velocities. This second layer also is much thicker than the boundary layer.

Considering these results a few remarks can be made. In the first place the question of stability arises: would it be possible to construct the solution as a Cauchy problem in time? A definite answer can not be given as yet. But there is one strong indication for stability namely that these solutions can be calculated iteratively by means of the difference method. It would of course also be interesting to design experiments in order to prove that these second solutions do exist indeed in physical reality.

The last remark is that the existence proof as given by McLeod for $s>0$ is not valid for the second branch since the basic assumption in the proof i.e. $g(x)>0$, is violated.

\subsection{Comparison with previous investigations}

Rogers and Lance [1] and Evans [2] also have obtained results for the problem with zero suction. In all cases they present, the range of integration is $0 \leqq x \leqq 12$ which as will be clear from our investigations leads inevitably to inaccurate results for negative values of $s$. A comparison is made in Table 4.5 which underlines this conclusion. Evans also discussed the case with suction. It will be evident, that our methods are also very suited to deal with this problem, although we have not considered this case here.

In [15], Bodonyi gives a time dependent method which he also uses to find results for stationary problems. Results of this kind are included in [4] as well. Since his method is a difference method of an order of accuracy in the stepsize comparable to that of Section 3.1, his results are only accurate in about 3 digits. Moreover as we have made clear, such a method is, at least in its present shape, unable to resolve the situation near a branching point. Therefore the question, whether or not, the occurrence of limit cycles is a basic phenomenon in the time dependent case, seems to be worthy of a reinvestigation.

A special case to give attention to is furnished by a paper of Weidman and Redekopp [5], 
TABLE 4.5

Comparison with the results of Rogers and Lance [1] and Evans [2].

\begin{tabular}{|c|c|c|c|c|}
\hline \multirow[b]{2}{*}{$s$} & \multicolumn{2}{|c|}{ Rogers and Lance } & \multicolumn{2}{|c|}{ Present results } \\
\hline & $2 f(\infty)$ & $-g^{\prime}(0)$ & $2 f(\infty)$ & $-g^{\prime}(0)$ \\
\hline 0.9 & 0.10201 & 0.096951 & 0.10200741 & 0.09695077 \\
\hline 0.8 & 0.20801 & 0.187591 & 0.20800654 & 0.18759077 \\
\hline 0.6 & 0.43077 & 0.348434 & 0.43077148 & 0.34843403 \\
\hline 0.4 & 0.65996 & 0.478673 & 0.65998069 & 0.47867327 \\
\hline 0.2 & 0.86175 & 0.572080 & 0.86169898 & 0.57208036 \\
\hline 0.1 & 0.91769 & 0.601554 & 0.91761139 & 0.60155285 \\
\hline 0 & 0.88446 & 0.615922 & 0.88447411 & 0.61592201 \\
\hline-0.05 & - & 0.615676 & 0.79413093 & 0.61565919 \\
\hline \multirow[t]{2}{*}{-0.10} & - & 0.608253 & 0.59450856 & 0.60828605 \\
\hline & \multicolumn{2}{|l|}{ Evans } & \multicolumn{2}{|c|}{ Present Results } \\
\hline$-s$ & $f^{\prime \prime}(0)$ & $-g^{\prime}(0)$ & $f^{\prime \prime}(0)$ & $-g^{\prime}(0)$ \\
\hline 0 & 0.51022912 & 0.61591916 & 0.51023262 & 0.61592201 \\
\hline 0.1 & 0.49130550 & 0.60825056 & 0.49128369 & 0.60828605 \\
\hline 0.15 & 0.47627301 & 0.58761507 & 0.47636622 & 0.58746927 \\
\hline 0.16 & 0.47332988 & 0.57766748 & 0.47353360 & 0.57600559 \\
\hline
\end{tabular}

presented at the Biennial Fluid Dynamics Symposium 1975 in Poland. They calculate a series expansion for the quantities $f_{\infty}, f^{\prime \prime}(0)$ and $g^{\prime}(0)$ in $s$ about $s=0$. Then they use the so called Domb-Sykes plot to investigate the behaviour of the singularity of the series expansions. That means that they plot the ratio of succeeding coefficients $c_{n}$ as a function of $1 / n$ and conclude from their results that there should hold approximately

$$
\begin{gathered}
f_{\infty}=-21.818205(s+0.1433)^{\frac{4}{3}}+2.0783525+15.788395 s \\
+12.2026 s^{2}-6.934 s^{3}+4.4095 s^{4}+0.53 s^{5} \\
f^{\prime \prime}(0)=0.206226(s+0.1433)^{\frac{4}{3}}+0.494768-0.034579 s \\
\quad-0.96315 s^{2}+0.4186 s^{3}-0.256 s^{4}+0.079 s^{5} \\
g^{\prime}(0)=1.263029(s+0.1433)^{\frac{4}{3}}-0.710635-0.82963 s \\
+0.01944 s^{2}+0.0115 s^{3}+0.003 s^{4}-0.007 s^{5}
\end{gathered}
$$

It will be quite clear that they infer from their results, that there is a singularity with a $\frac{4}{3}$ power and that this singularity occurs approximately for $s=-0.1433$. They propose that inclusion of more terms in the series expansion should shift the location of the singularity to $s=-0.154$, the point where $f_{\infty}$ vanishes. According to our results these conclusions are wrong in the sense, that neither the type nor the place of the singularity are correct. On the other hand, there is no doubt that the numerical results Weidman and Redekopp present for the series expansion around $s=0$ are accurate. What then went wrong? 
The reason can be seen by considering for instance eq. (4.2). It follows from this equation that there should hold

$$
f_{\infty}=1.402(s+0.1605387613)^{\frac{1}{2}}+\sum_{i=0} c_{i} s^{i}{ }^{\star}
$$

Now using the values Weidman and Redekopp calculated, it is easy to find the first coefficients $c_{i}$. It turns out that there should hold

$$
\begin{aligned}
f_{\infty}= & 1.402(s+0.1605387613)^{\frac{1}{2}}-0.11951-1.1844 s-2.7784 s^{2} \\
& +12.037 s^{3}-42.390 s^{4}+153.62 s^{5}-594.06 s^{6}+2456.5 s^{7}-10831 s^{8} .
\end{aligned}
$$

We now calculate the value of $f_{\infty}$ for $s=-0.1605387613$. It turns out that according to eq. (4.9) $f_{\infty}=-0.11702$ whereas according to eq. (4.2) the correct value should be $f_{\infty}=-0.13718$. This means that even the corrected series is way off of the correct result, and that we should need much more terms to get the correct results. But then it should be clear that an analysis of the singular behaviour based on the first terms can lead and in this case actually does lead to wrong results. As can be seen from Figs. $4 \mathrm{a}$ and $4 \mathrm{~b}$ the singular behaviour for $f^{\prime \prime}(0)$ and $g^{\prime}(0)$ also is very locally, which for these cases too, leads to the expectation that many terms more in the series are needed to predict adequately the right behaviour. This then at the same time should yield a serious warning, when using the Domb-Sykes plot, to make oneself sure that the power of the singularity and its location have settled down.

\section{Conclusions}

In the course of our investigations on the problem of the counterrotating fluid above a flat rotating plate, we have shown that this problem (which is governed by the full Navier-Stokes equations) is non-unique. Effective numerical methods have been devised to deal with such a situation. We have calculated the second branch and we feel that these solutions exhibit peculiarities which are extremely interesting to all those working in this field, be it on subjects of meteorology or of oceanography or of geophysics. We believe that these methods can also be used for other interesting problems related to rotating flow, for instance the vortex source flow as treated by Cham [16], or the problem of two disks.

A number of interesting problems remains to be solved. Is it possible to give existence proofs for the new solutions? Can these solutions also be found as the stationary solution of a Cauchy problem, i.e. are they stable in a mathematical sense? As we have said, we believe they are. Thus the next question is to show experimentally that such solutions occur or may occur. Another point is the construction of the rest of the solution branch. We think that the curve ultimately will go to the point $f_{\infty}=0, s=0$, but more analytical work will be needed to clarify the situation.

\footnotetext{
* Note added in proof. It will be clear that (4.8) is not an expansion for $s \rightarrow s_{c r}$. It should be regarded as some kind of approximation identical to the Weidman and Redekopp approach and set up to demonstrate the failure of this approach.
} 


\section{Acknowledgement}

The authors want to thank Mr. E. H. Derks for performing a large part of the calculations.

\section{Appendix. First-order perturbation theory near the critical point}

In this section we present further analytical background concerning the behaviour of the solution near the critical point $s_{c r}$. From the numerical results it is already clear that the solution has a square root singularity in terms of $s$ at the critical point. Therefore, we could set up an expansion of the following form

$$
f=f_{0}+\sqrt{s-s_{c r}} f_{1}+\ldots, s \rightarrow s_{c r}
$$

Together with $g$, this leads to an additional set of differential equations for $f_{1}$ and $g_{1}$ which requires numerical integration. Since numerical methods with $s$ prescribed are doomed to fail near $s_{c r}$, we discard expansion (A.1) and set up an expansion in terms of the formulation (2.12)(2.14) with $F(\infty)=A$ as the independent parameter. Near the critical point $A_{c r}$, all quantities vary linearly with $A$, except $s$ which varies quadratically (see Tables $4.3 \mathrm{~b}$ and $4.3 \mathrm{c}$ ). The expansion in terms of $A$ is regular and can also be used for points $A_{0}$ different from $A_{c r}$. This is another advantage of this approach as compared to (A.1). The expansions are taken as

$$
F=F_{0}+\left(A-A_{0}\right) F_{1}+\ldots, G=G_{0}+\left(A-A_{0}\right) G_{1}+\ldots, A=F(\infty) .
$$

The functions $F_{0}$ and $G_{0}$ satisfy the original problem (2.12)-(2.14) with $F_{0}(\infty)=A_{0}$ prescribed. The equations governing the perturbations $F_{1}$ and $G_{1}$ can be obtained by substituting (A.2) in (2.12)-(2.14). Equivalently, we can differentiate the problem with respect to $A$. The full set of resulting equations reads

$$
\begin{aligned}
& F_{0}^{\prime \prime \prime}+2 F_{0} F_{0}^{\prime \prime}-F_{0}^{\prime 2}+G_{0}^{2}=1, \\
& G_{0}^{\prime \prime}+2 F_{0} G_{0}^{\prime}-2 F_{0}^{\prime} G_{0}=0 \\
& F_{1}^{\prime \prime \prime}+2 F_{0} F_{1}^{\prime \prime}-2 F_{0}^{\prime} F_{1}^{\prime}+2 F_{0}^{\prime \prime} F_{1}+2 G_{0} G_{1}=0, \\
& G_{1}^{\prime \prime}+2 F_{0} G_{1}^{\prime}-2 F_{0}^{\prime} G_{1}+2 G_{0}^{\prime} F_{1}-2 G_{0} F_{1}^{\prime}=0 .
\end{aligned}
$$

The boundary conditions are

$$
\begin{aligned}
& F_{0}(\infty)=A_{0}, F_{0}^{\prime}(\infty)=0, G_{0}(\infty)=1, F_{0}(0)=0, F_{0}^{\prime}(0)=0, \\
& F_{1}(\infty)=1, F_{1}^{\prime}(\infty)=0, G_{1}(\infty)=0, F_{1}(0)=0, F_{1}^{\prime}(0)=0 .
\end{aligned}
$$

Note that the equations governing $F_{1}$ and $G_{1}$ are linear and homogeneous. The solutions $F_{1}$ and $G_{1}$ are induced by the condition $F_{1}(\infty)=1$. Further, the quantity $s$ does not appear in the problem. The parameter $s$ is an output of the calculation, viz.

$$
s_{0}=1 / G_{0}(0)
$$

see eq. (2.14).

In addition we now have an analytical expression for the derivative $d s / d A$. This is given by

$$
\left(\frac{d s}{d A}\right)_{0}=-s_{0}^{2} G_{1}(0) .
$$


At the critical point the quantity $s$ is stationary hence

$$
A_{0}=A_{c r} \Rightarrow s_{0}=s_{c r}, \quad\left(\frac{d s}{d A}\right)_{0}=0 .
$$

In other words, the critical point is determined through the additional condition

$$
G_{1}(0)=0
$$

The numerical method to find the critical point is obvious: solve the system (A.3)-(A.6) in the neighbourhood of the critical point and then iterate on $A_{0}$ until (A.10) is satisfied. To solve (A.4) by inverse shooting as described in Sec. 3.2, we need the asymptotic behaviour for large $\eta$ of the functions $F_{1}$ and $G_{1}$. To first order this follows from the equations

$$
\begin{aligned}
& \bar{F}_{1}^{\prime \prime \prime}+2 A_{0} \bar{F}_{1}^{\prime \prime}+2 \bar{G}_{1}=-2 \bar{F}_{0}^{\prime \prime}, \\
& \bar{G}_{1}^{\prime \prime}+2 A_{0} \bar{G}_{1}^{\prime}-2 \bar{F}_{1}^{\prime}=-2 \bar{G}_{0}^{\prime},
\end{aligned}
$$

where $\bar{F}_{0}^{\prime \prime}$ and $\bar{G}_{0}^{\prime}$ are first-order asymptotic expressions which can be found from eqs. (2.15) and (2.16). The solution of (A.11) is found to be $\left(\bar{F}_{1}^{\prime}\right.$ only)

$$
\bar{F}_{1}^{\prime}=\eta \mathrm{e}^{P_{0} \eta}\left(\bar{B}_{0} \sin Q_{0} \eta+\bar{C}_{0} \cos Q_{0} \eta\right)+\mathrm{e}^{P_{0} \eta}\left(B_{1} \sin Q_{0} \eta+C_{1} \cos Q_{0} \eta\right)
$$

where

$$
\begin{aligned}
& \bar{B}_{0}=-B_{0} \frac{P_{0}^{2}+A_{0} P_{0}+Q_{0}^{2}}{\left(P_{0}+A_{0}\right)^{2}+Q_{0}^{2}}+C_{0} \frac{A_{0} Q_{0}}{\left(P_{0}+A_{0}\right)^{2}+Q_{0}^{2}}, \\
& \bar{C}_{0}=-B_{0} \frac{A_{0} Q_{0}}{\left(P_{0}+A_{0}\right)^{2}+Q_{0}^{2}}-C_{0} \frac{P_{0}^{2}+A_{0} P_{0}+Q_{0}^{2}}{\left(P_{0}+A_{0}\right)^{2}+Q_{0}^{2}} .
\end{aligned}
$$

Another interesting method to obtain this result is the following. The first-order asymptotic approximation to $F^{\prime}$ for large $\eta$ follows from (2.15) and is given by

$$
F^{\prime} \sim \mathrm{e}^{P \eta}(B \sin Q \eta+C \cos Q \eta)
$$

Differentiating this with respect to $A$ we obtain

$$
\begin{aligned}
\frac{d F^{\prime}}{d A} & \sim \eta \mathrm{e}^{P \eta}\left\{\left(B \frac{d P}{d A}-C \frac{d Q}{d A}\right) \sin Q \eta+\left(B \frac{d Q}{d A}+C \frac{d P}{d A}\right) \cos Q \eta\right\} \\
& +\mathrm{e}^{P \eta}\left(\frac{d B}{d A} \sin Q \eta+\frac{d C}{d A} \cos Q \eta\right) .
\end{aligned}
$$

Calculating the derivatives from (2.17) and using (A.2) we obtain (A.12). In other words the asymptotic behaviour of the perturbation is equal to the perturbation of the asymptotic approximation. A further consequence of (A.14) is 


$$
B_{1}=\left(\frac{d B}{d A}\right)_{0}, \quad C_{1}=\left(\frac{d C}{d A}\right)_{0}
$$

which can be used as a check on the numerical results.

We observe that the first term in the result (A.12) is a particular solution of (A.11), and that the second term is a solution of the homogeneous problem. The two degrees of freedom in the homogeneous solution can be used to satisfy the boundary conditions (A.6) at the origin.

The numerical method to solve the critical problem is now briefly described. We select an interval in which the critical point is contained. From Table (4.3b) this is taken as

$$
-0.345 \leqq A_{0} \leqq-0.340
$$

With $A_{0}=-0.345(0.0005)-0.340$, the problem (A.3) with boundary conditions (A.5) is solved using the method described in Section 3.2. This is done with an accuracy of $\approx 10$ digits. With the resulting values for the asymptotic parameters $B_{0}$ and $C_{0}$, the quantities $\bar{B}_{0}$ and $\bar{C}_{0}$ are calculated from (A.13).

At this stage, the first-order asymptotic approximation (A.12) is known, except for the constants $B_{1}$ and $C_{1}$. Next, the full problem (A.3)-(A.6) is solved in the same points $A_{0}$ as above. The only iteration in the full problem is one on $B_{1}$ and $C_{1}$ (to satisfy (A.6) at the origin), since $B_{0}$ and $C_{0}$ have been calculated already in the first stage, so that the conditions (A.5) at the origin are automatically satisfied. We observe that one iteration step on $B_{1}$ and $C_{1}$ is sufficient since the problem (A.4) is linear.

The results of the calculation are tabulated in Tables $4.4 \mathrm{a}$ and $4.4 \mathrm{~b}$ and graphically represented in Figs. 5a and $5 b$.

\section{REFERENCES}

[1] M. H. Rogers and G. N. Lance, The rotationally symmetric flow of a viscous fluid in the presence of an infinite rotating disk. Journal of Fluid Mechanics, 7 (1960) 617-631.

[2] D. J. Evans, The rotationally symmetric flow of a viscous fluid in the presence of an infinite rotating disk with uniform suction. Quarterly Journal of Mechanics and Applied Mathematics, 22 (1969) 467-485.

[3] H. Ockendon, An asymptotic solution for steady flow above an infinite rotating disk with suction. Quarterly Journal of Mechanics and Applied Mathematics, 25 (1972) 291-301.

[4] R. J. Bodonyi, On rotationally symmetric flow above an infinite rotating disk. Journal of Fluid Mechanics, 67 (1975) 657-666.

[5] P. D. Weidman and L. G. Redekopp, On the motion of a rotating fluid in the presence of an infinite rotating disk. Lecture presented at the 12th Biennial Fluid Dynamics Symposium, september 1975, Bialowieza, Poland.

[6] S. P. Hastings, An existence theorem for some problems from boundary layer theory. Archive for Rational Mechanics and Analysis, 38 (1970) 308-316.

[7] J. B. McLeod, The asymptotic form of solutions of Von Karman's swirling flow problem. Quarterly Journal of Mathematics (Oxford), 20 (1969) 483-496.

[8] J. B. McLeod, The existence of axially symmetric flow above a rotating disk. Proceedings of the Royal Society, A 324 (1971) $391-414$.

[9] J. B. McLeod, A note on rotationally symmetric flow above an infinite rotating disk. Mathematika, 17 (1970)243249.

[10] P. J. Bushell, On Von Karman's equations of swirling flow. Journal of the London Mathematical Society (2), 4 (1972) 701-710.

[11] P. Hartman, On the swirling flow problem. Indiana University Mathematical Journal, 21 (1972) 849-855. 
[12] C. C. Lan, On functional differential equations and some laminar boundary layer problems. Archive for Rational Mechanics and Analysis, 42 (1971) $24-39$.

[13] H. Schlichting, Boundary-layer theory. McGraw-Hill, New York (1968).

[14] R. Bulirsch and S. Stoer, Numerical treatment of ordinary differential equations by extrapolation methods. Numerische Mathematik, 8 (1966) 1-13,93-104.

[15] R. J. Bodonyi, The laminar boundary layer on a finite rotating disc. Dissertation, Dept. of Aeronautical and Astronautical Engineering, The Ohio State University (1973).

[16] T. S. Cham, The laminar boundary layer of a source and vortex flow. The Aeronautical Quarterly, May 1971, 196206. 